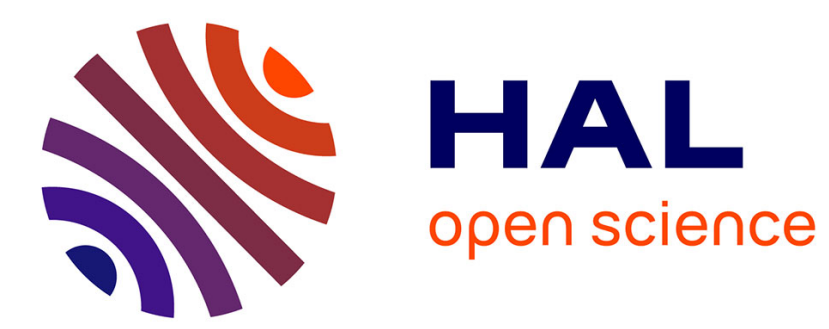

\title{
Tracer Studies on F-Actin Fluctuations
}

\author{
Loïc Le Goff, Oskar Hallatschek, Erwin Frey, François Amblard
}

\section{To cite this version:}

Loïc Le Goff, Oskar Hallatschek, Erwin Frey, François Amblard. Tracer Studies on F-Actin Fluctuations. Physical Review Letters, 2002, 89 (25), pp.258101. 10.1103/physrevlett.89.258101 . hal02415551

\section{HAL Id: hal-02415551 \\ https://hal.science/hal-02415551}

Submitted on 19 Dec 2019

HAL is a multi-disciplinary open access archive for the deposit and dissemination of scientific research documents, whether they are published or not. The documents may come from teaching and research institutions in France or abroad, or from public or private research centers.
L'archive ouverte pluridisciplinaire HAL, est destinée au dépôt et à la diffusion de documents scientifiques de niveau recherche, publiés ou non, émanant des établissements d'enseignement et de recherche français ou étrangers, des laboratoires publics ou privés. 


\title{
Tracer Studies on F-Actin Fluctuations
}

\author{
Loïc Le Goff, ${ }^{1, *}$ Oskar Hallatschek, ${ }^{2}$ Erwin Frey, ${ }^{2,3}$ and François Amblard ${ }^{1}$ \\ ${ }^{1}$ Institut Curie, Physico-Chimie Curie, UMR CNRS/IC 168, 26 rue d'Ulm, 75248 Paris Cedex 05, France \\ ${ }^{2}$ Abteilung Theorie, Hahn-Meitner-Institut, Glienicker Strasse 100, 14109 Berlin, Germany \\ ${ }^{3}$ Fachbereich Physik, Freie Universität Berlin, Arnimallee 14, 14195 Berlin, Germany
}

\begin{abstract}
We present a study on the fluctuations of semiflexible actin filaments using fluorescence videomicroscopy, focusing on the end-to-end fluctuations of single filaments. In order to specifically measure the position of the polymer's ends, we developed a novel noninvasive method that consists of annealing short end tags to the filaments. This allows us to probe polymer fluctuations to a very high accuracy. We compared the distribution of the end-to-end distance with recent theoretical results, and found excellent agreement. We also studied the dynamics of the mean-square end-to-end distance $\delta R^{2}(t)$ and orientation of the ends, $\delta \Theta^{2}(t)$, finding power laws $t^{3 / 4}$ and $t^{1 / 4}$, respectively. Scaling behavior for $\delta R^{2}(t)$ is observed over several decades in relaxation time in agreement with theoretical results.
\end{abstract}

Actin filaments have been the subject of intensive biophysical in vitro studies over the past years. Besides the motivation to mimic cellular mechanics with reconstituted purified systems, actin, one of the few available semiflexible polymers, became a model case to study the effect of rigidity on polymer dynamics. In addition to bulk rheological measurement [1], recent advances in the understanding of the equilibrium or dynamic properties of actin solutions have required the direct visualization of actin filaments [2-4], or the use of tracer particles imbedded in a dense network to measure dynamic shear modulus [5-7]. A key problem in the theory of viscoelasticity [8] is the bridging of information from these two different scales, rheological response, and single filament dynamics. In general, this is an unsolved manychain problem involving a subtle interplay between steric interactions and viscous coupling between the filaments. Only for high frequencies, theories assuming that individual filaments affinely follow external shear deformations have successfully linked the time dependence of the mean-square displacement $\delta R^{2}(t) \equiv\left\langle[R(t)-R(0)]^{2}\right\rangle$ of the end-to-end distance $R$ to the frequency dependence of the macroscopic shear modulus $[9,10]$.

Another important quantity for understanding the elastic plateau of a solution is the probability distribution function $G(R)$ of the end-to-end distance for individual filaments [11]. For chains at least 10 times longer than their persistence length, a Gaussian distribution can serve as an excellent approximation. The well-known minimal model of flexible polymers, the Gaussian chain, is obtained by imposing that this Gaussian behavior is valid down to arbitrarily short length scales. Polymers that are shorter or comparable to their persistence length show a very different end-to-end behavior. With increasing stiffness, there is a pronounced crossover from a Gaussian to a completely non-Gaussian distribution with the function weighted towards full stretching. For a semiflexible poly- mer constrained to fluctuate in two dimensions, an explicit expression for the distribution function can be found in Refs. [11,12].

It would be crucial for our understanding of semiflexible polymer dynamics and viscoelasticity to assess both $G(R)$ and $\delta R^{2}(t)$ with great accuracy. It is the aim of this Letter to show that one can probe both of these quantities in one single measurement, improving in that way previous single filament assays which focused only on static properties [2,3] and center of mass motion [4]. For this purpose, we developed a novel noninvasive method based on annealing polymerization, and by which the filament is tagged only at its ends. As opposed to existing techniques based on whole filament fluorescent labeling or microsphere tags, our method leaves most of the chain free of any modification that could alter its mechanical or hydrodynamic properties. Speckle imaging [13] is also based on assessing fluorescent tag dynamics, but with a random tag distribution.

Actin is known to polymerize under physiological conditions following a nucleation elongation process [14] for which all rate constants have been fully characterized [15]. It has also been established that filaments can break and anneal end-to-end [14,16]. More recently, clear evidence was given that annealing is a driving mechanism in controlling the length distribution [17]. Based on these observations, we mixed sparsely fluorescently labeled filaments with densely labeled ones, yielding in typical "copolymer" filaments as shown in Fig. 1. The size of the brighter segments was reduced to below optical resolution by gentle sonication. Their position and orientation was then easily determined by image analysis, and thereby they acted as end tags for the darker filament. Our method, entirely based on fluorescence microscopy, relies on using these tracer fragments to probe the position of particular points locally on individual filaments. 


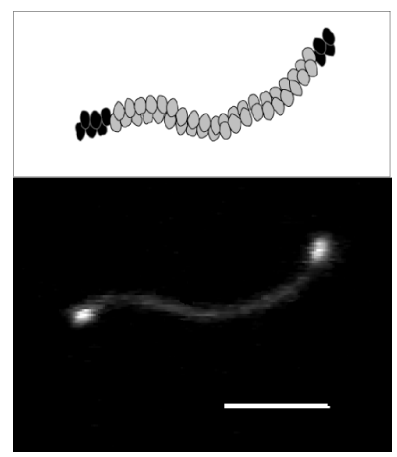

FIG. 1. Fluorescence microscopy image of an actin filament. As shown on the sketch, it is made of one long filament that annealed by the ends with two bright fragments. The scale bar is $5 \mu \mathrm{m}$. Monomers are not at scale on the sketch. The end tags are constituted of $\sim 50-100$ monomers.

In this Letter, we present the first experimental measurement of the projected length of actin filaments, with enough statistics and resolution to compute the radial distribution function and projected length dynamics. Both of these quantities have been the subject of theoretical works that could not, until now, be assessed experimentally. We first present results on the radial distribution function and compare it to the analytical expression of Ref. [11]. We then focus on dynamics, emphasizing how different mean-square displacements of the end-to-end distance can reduce to a single master curve. We conclude with remarks on angular bending dynamics.

Experimental procedure.-Actin is the same as in Ref. [18]. Alexa-568 labeled actin is purchased from Molecular Probes. All proteins are stored in $G$ buffer [2 mM Imidazole ( $\mathrm{pH}$ 7.5), $0.5 \mathrm{mM}$ ATP, $0.2 \mathrm{mM}$ $\mathrm{CaCl} 2,0.2 \mathrm{mM}$ DTT, $0.01 \% \mathrm{NaN} 3)]$ at a concentration of $1 \mathrm{mg} / \mathrm{ml}$ at $-80^{\circ} \mathrm{C}$. Annealed filaments with end tags are prepared as follows: to a $3 \mu \mathrm{M}$ solution of weakly fluorescent filaments (1/4 of monomers are labeled) in $F$ buffer $(25 \mathrm{mM}$ Imidazole, $2 \mathrm{mM} \mathrm{MgCl} 2,0.5 \mathrm{mM}$ ATP, $50 \mathrm{mM} \mathrm{KCl}, 0.5 \mathrm{mM}$ DTT, $0.01 \% \mathrm{NaN} 3$ ), we add 1/20 molar ratio of fully labeled end tags which have previously been shortened by bath sonication $\left(2-3\right.$ min at $4{ }^{\circ} \mathrm{C}$, under argon atmosphere). The density of fluorophores on the dimly fluorescent filaments is such that the labeling still appears homogeneous. Filaments and end tags are stabilized with an excess of phalloidin and allowed to spontaneously anneal for $1 \mathrm{~d}$ at $4{ }^{\circ} \mathrm{C}$. Single filament observation procedures are presented elsewhere [18]. Images are acquired with an intensified CCD camera (Lhesa), into which a cooling circuitry $\left(-20^{\circ} \mathrm{C}\right)$ has been added to reduce thermal noise. Actin monomers are covalently labeled with a stochiometry higher than two fluorophores per monomers, allowing the visualization of both the end tags and the darker filament for more than $30 \mathrm{~nm}$. Filaments are observed in a $1 \mu \mathrm{m}$ thick BSA-coated chamber, confining them into the focal plane of the microscope. Image analysis is performed in small regions centered around each tag, in which a threshold is automatically computed to distinguish it from the rest of the filament. The position of each tag is obtained by a centroid analysis with a resolution of $50 \mathrm{~nm}$ limited by photon shot noise.

Radial distribution function $(R D F)$. - We have tracked the end-to-end fluctuations of individual filaments. As described above, the movement is constrained to a $1 \mu \mathrm{m}$ deep volume, making the dynamics of the filaments essentially 2D. The observation time for each filament is limited by the photodamage of the bound fluorophores that tends to break filaments. We could thoroughly sample the configurational space of all filaments except the largest, since relaxation time increases rapidly with filament length $\left(\tau \propto L^{4}\right)$. Figure 2 shows a typical probability distribution $G(R)$ for the end-to-end distance $R$ of an actin filament sampled over 6200 configurations. It exhibits both of the characteristic features expected for semiflexible polymers. At small $R$, we find a decay $G(R) \propto$ $\exp \left[-f_{c}(L-R)\right]$ resulting from the energetic costs of buckling a classical beam with bending stiffness $\kappa$, where $f_{c}=\kappa \pi^{2} / L^{2}$ is the critical force for the onset of the Euler buckling instability. Approaching full stretching, $R \rightarrow L$, the number of allowed configurations reduces drastically, resulting in a sharp decay with $G(R) \propto$ $\exp [-1 / 4(L-R)] /(L-R)^{5 / 2}$. Comparison between the experimental RDF and the analytical expression of Eq. 5 in Refs. [11,12] shows excellent agreement. Adjusting the curves, by least mean square, with the two fitting parameters persistence length, $\ell_{p}=\kappa / k_{B} T$, and contour length, $L$, we obtain $\ell_{p}=16.1 \pm 0.2 \mu \mathrm{m}$ and $L=$ $13.40 \pm 0.01 \mu \mathrm{m}$ (error bars are determined by MonteCarlo method). Because the dark filament can still clearly be distinguished, we can separately estimate $\ell_{p}$ and $L$ by a tracing algorithm described elsewhere [18]. The persistence length is extracted from the tangent-tangent

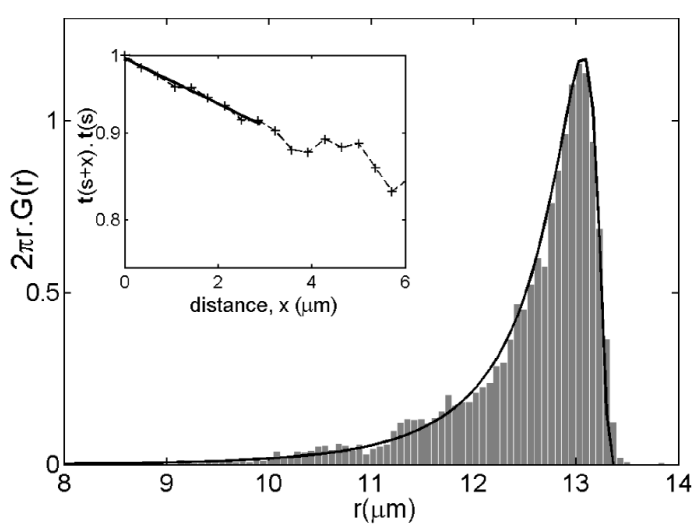

FIG. 2. Measured radial distribution function (bars), and theoretical expression for 2D RDF (line) with $\ell_{p}=16.1 \pm$ $0.2 \mu \mathrm{m}$ and $L_{c}=13.40 \pm 0.01 \mu \mathrm{m}$. Inset: Measured tangent correlation function of the filament $(-+-)$, and single exponential decay adjustment $\mathbf{t}(s+x) \cdot \mathbf{t}(s)=e^{-x / 2 l_{p}} \quad$ (thick line), yielding a persistence length $l_{p}=16.2 \pm 0.7 \mu \mathrm{m}$. 
correlation function (see the inset of Fig. 2), which decays as a single exponential [2]. We obtain $\ell_{p}=16.2 \pm 0.7 \mu \mathrm{m}$ and $L=13.5 \pm 0.3 \mu \mathrm{m}$. As expected, the estimate of $L$ and $l_{p}$ from the RDF is more accurate than from the contour analysis. This latter method can easily give rise to a pixelization roughening at small scales, while the position of ovoidal tracers can be computed with subpixel accuracy.

Projected length dynamics. - An important observable for characterizing the dynamics of semiflexible polymers is the time-dependent mean-square difference (MSD) of the end-to-end distance, $\delta R^{2}(t) \equiv\langle[R(t)-$ $\left.R(0)]^{2}\right\rangle$. We estimated this MSD by replacing the ensemble average of the brackets with a time average, scanning over initial times $t_{0}, \delta R^{2}(t)=\left\langle\left[R\left(t_{0}+t\right)-\right.\right.$ $\left.\left.R\left(t_{0}\right)\right]^{2}\right\rangle_{t_{0}}$. The corresponding data sets are shown in the inset of Fig. 3.

We model the dynamics of a semiflexible filament in terms of a linear Langevin equation in the free draining limit with an effective friction coefficient $\zeta$ [19]. Then the MSD should obey a scaling law of the form $\delta R^{2}(L, t)=$ $L^{4} F\left(t / \tau_{c}\right) /\left(90 \ell_{p}^{2}\right)$.

Here $\tau_{c} \propto(\zeta / \kappa) L^{4}$ is a characteristic time scale where the end-to-end fluctuations begin to saturate at their corresponding equilibrium value $\delta R_{\mathrm{eq}}^{2}=L^{4} /\left(90 \ell_{p}^{2}\right)$. These universal scaling features can be seen in Fig. 3, where we have scaled the magnitude of the MSD by the equilibrium value and the time by the characteristic crossover time. We find a remarkably good agreement with the theoretical scaling function (solid line) over several orders of magnitude in rescaled times.

Upon performing a normal mode analysis, the scaling function $F$ can be written in terms of the eigenvectors of the bending modes of the filament. For free ends, the

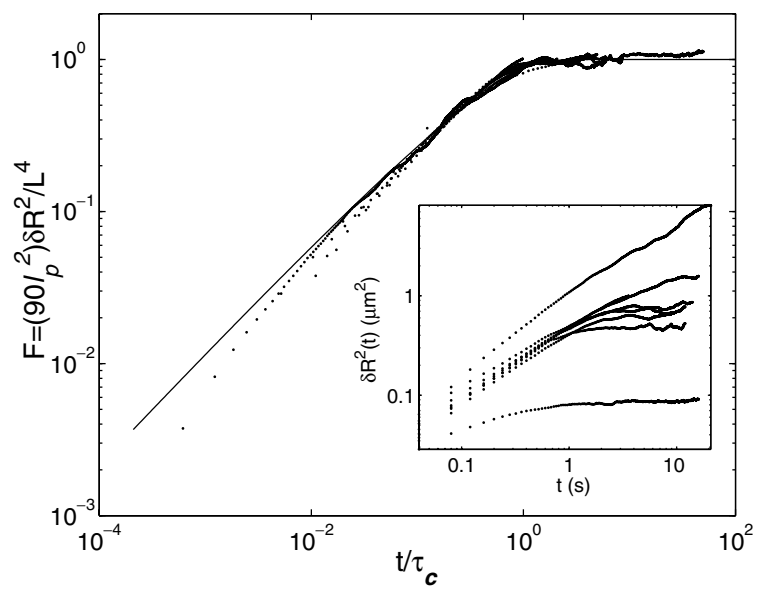

FIG. 3. Rescaled MSD $F=\left(90 \ell_{p}^{2}\right) \delta R^{2}(L, t) / L^{4}$ as a function of rescaled time $t / \tau_{c}=t(4.73 / L)^{4}(\kappa / \zeta)$; the solid line is the prediction for a weakly bending rod in two dimensions. Inset: Raw data before rescaling for filaments with lengths $L[\mu \mathrm{m}]=$ $\{5.9$ (bottom), 10.4, 10.7, 11.8, 11.8, 11.9, 12.9, 25.6 (top)\}. The curve results from the analysis of over 60000 images. mode spectrum is determined by $\cos k_{n}=1 / \cosh k_{n}$, and the eigenmodes are given by biharmonic functions [20]. Because the resulting analytic expression for the MSD is too long, we restricted our discussion to the limiting cases. For times much smaller than the longest relaxation time $\tau_{c}=(\zeta / \kappa)(L / A)^{4}$ with $A \approx 4.73$, one gets a simple power law,

$$
\delta R^{2}(L, t)=B \frac{L}{l_{p}^{2}}\left(\frac{t \kappa}{\zeta}\right)^{3 / 4},
$$

with $B \approx 2.71$. For $t \gg \tau_{c}$, the MSD saturates at a value of $\delta R_{\text {eq }}^{2}$, which as an equilibrium quantity is independent of the friction coefficient. We would like to emphasize that both the crossover time $\tau_{c}$ and the amplitude of the power law, Eq. (1), depend on the boundary conditions of the problem. For example, a filament with hinged ends $[9,10,19]$ takes 5 times longer to relax than a free one ( $A_{\text {hinged }}=\pi, B_{\text {hinged }}=0.647$ ). We now choose the persistence length and the friction coefficient such that the theory fits the data in the saturated and in the power law regime, respectively. We find the values $l_{p}=15.7 \pm$ $1 \mu \mathrm{m}$ and $\zeta=5.6 \pm 0.6 \times 10^{-3} \mathrm{Pas}$. The error bars reflect the filament to filament variation in elasticity, intrinsic to the actin preparation, and in the friction, setting the reproducibility limit of our in vitro assay. The value of $\zeta$ agrees well with the friction coefficient of a rigid rod between two glass plates [21], $\zeta_{\text {hydro }}=3.2 \times 10^{-3} \mathrm{Pas}$. The additional friction can be attributed to interactions with the coverslip surfaces.

A possible interference with our measurements comes from the fact that the experimental setup is actually not perfectly two dimensional. Indeed, the finite distance $d=1 \mu \mathrm{m}$ between the walls allows for some small outof-plane fluctuations that increase the MSD by an additional term $\delta R_{\perp}^{2}(t)$. Upon modeling the constraint by a harmonic potential, we found that for most filaments under consideration the effect of out-of-plane fluctuations to the MSD is smaller than the experimental error [23].

The above linear mode analysis neglects effects due to tension propagation along the filament. As pointed out in Ref. [24], this has dramatic consequences on the scaling behavior of the longitudinal fluctuations at the polymer's end. At short times a $t^{7 / 8}$ instead of a $t^{3 / 4}$ behavior was predicted [24], and indeed observed in recent experiments [18]. Here, in contrast to such a local quantity, we measure the dynamics on the global scale of the end-toend distance, where the dominant scaling always is $t^{3 / 4}$. Taking into account effects due to tension propagation would modify the above results slightly. Essentially, it would lead to corrections in the amplitude of the end-toend distance but not the scaling behavior. In the linear theory, contributions from longitudinal fluctuations are overestimated by roughly a factor of $\left[\tau_{c}(L) / t\right]^{1 / 8}$ for times smaller than $\tau_{c}$, the time for the tension to propagate over the full length of the filament. Probing such small effects is not feasible given the accuracy and statistics of the present approach. 


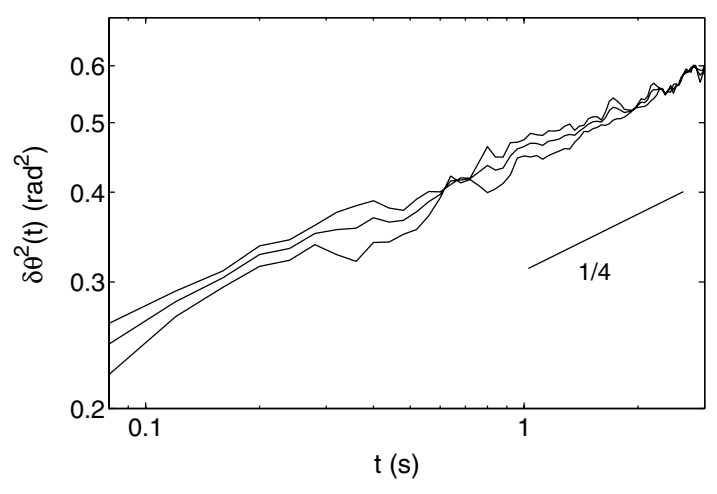

FIG. 4. Measured MSD for the angle of a tag, giving local orientational dynamics. The plot is logarithmic; $a \propto t^{1 / 4}$ guideline is represented.

Angular dynamics. - When the length of a tag is slightly larger than the optical resolution of the microscope, we can resolve its orientation, in addition to its position. Tracing the time fluctuations of its angle yields complementary information on the filament dynamics. Figure 4 shows the MSD of the angle of a tracer located on a $10 \mu \mathrm{m}$ long filament. It exhibits a $t^{1 / 4}$ power law, consistent with an equilibrium amplitude $\delta \theta^{2} \propto L / l_{p}$ and an equilibration length $l_{\perp} \propto\left(l_{p} t / \zeta\right)^{1 / 4}$ for transverse modes.

In this Letter, we reported on the first measurement of radial distribution function and projected length dynamics. For this, we developed a novel tracer method to determine the position and orientation of individual points on filaments, which does not require the addition of mechanically invasive objects such as colloidal probes. We found that the analytical expression previously published [11] provides an excellent fit of the experimental $\mathrm{RDF}$, indicating that the conformations of actin can be well characterized by only two parameters, its contour and persistence lengths. Our analysis shows that shortscale information (tangent correlation function) and large-scale information (RDF) converge to very close persistence length values. An experimental prerequisite for this scale-independent elasticity lies in a good actin preparation to avoid defects on filaments. We have also measured the dynamical fluctuations of the end-to-end vector. A single scaling function, whose form can be derived analytically, can describe the MSD of the endto-end distance, for filaments of all lengths over a wide range of time scales. Our tracer analysis is relevant to semidilute solution studies with the aim of linking microrheology and macrorheology. Also, phalloidin stabilized end tags can be used to probe the elasticity of unstabilized and unlabeled filaments. This can be a helpful feature for studying mechanical alteration of actin filaments induced by proteins whose binding is impeded by phalloidin derivatives.
We thank J. Wilhelm, E. M. Furst, and T. Liverpool for discussions, J. Mertz for critical reading of the manuscript, and S. Tsacas and J. Wilhelm for help in computer programming. Financial support from DGA (L. L. G.) and ARC (L. L. G.) and grants from CNRS-PCV and CuriePIC (F. A.) are gratefully acknowledged.

*Author to whom correspondence should be addressed. Electronic address: Loic.Legoff@curie.fr

[1] B. Hinner et al. Phys. Rev. Lett. 81, 2614 (1998).

[2] A. Ott et al., Phys. Rev. E 48, R1642 (1993).

[3] F. Gittes et al., J. Cell Biol. 120, 923 (1993).

[4] J. Käs, H. Strey, and E. Sackmann, Nature (London) 368, 226 (1994).

[5] F. Amblard et al., Phys. Rev. Lett. 77, 4470 (1996).

[6] F. Gittes et al., Phys. Rev. Lett. 79, 3286 (1997).

[7] T. Gisler and D. Weitz, Phys. Rev. Lett. 82, 1606 (1999).

[8] A. Caspi et al., Phys. Rev. Lett. 80, 1106 (1998).

[9] F. Gittes and F. C. MacKintosh, Phys. Rev. E 58, R1241 (1998).

[10] D. C. Morse, Macromolecules 31, 7030 (1998); 31, 7044 (1998).

[11] J. Wilhem and E. Frey, Phys. Rev. Lett. 77, 2581 (1996).

[12] For the 2D RDF the only point where the harmonic approximation enters the calculation is at the evaluation of the end-to-end distance [11]. Effectively, the actual end-to-end distance is replaced by a distribution of end-to-end points. Some of these deviations can be corrected for by calculating the difference in the exact and approximate end-to-end distance for the classical problem. This gives an improved expression of Eq. 5 in Ref. [11], where $x=1-R$ is replaced by $\Delta(x) \approx x-$ $0.22 x^{2}+0.011 x^{4}$.

[13] C. M. Waterman-Storer et al., Curr. Biol. 8, 1227 (1998).

[14] F. Oosawa and S. Asakura, Thermodynamics of the Polymerization of Protein (Academic, New York, 1975).

[15] M. F. Carlier, J. Biol. Chem. 266, 1 (1991).

[16] D. B. Murphy et al., J. Cell Biol. 106, 1947 (1988).

[17] D. Sept et al., Biophys. J. 77, 2911 (1999).

[18] L. Le Goff, F. Amblard, and E. M. Furst, Phys. Rev. Lett. 88, 018101 (2002)

[19] R. Granek, J. Phys. II (France) 7, 1761 (1997).

[20] Ch. Wiggins et al., Biophys. J. 74, 1043 (1998).

[21] A rod of diameter $a$ between walls of distance $d=1 \mu \mathrm{m}$ has an effective friction coefficient of [22] $\zeta \simeq(4 \pi \eta) /$ $[\ln (d / 2 a)-0.9157] \approx 3.2 \times 10^{-3} \mathrm{~Pa} \mathrm{~s}$, where $\eta$ is the viscosity.

[22] J. Happel and H. Brenner, Low Reynolds Number Hydrodynamics (Kluwer, Dordrecht, 1983).

[23] Typically, their relative contribution to the saturation value of the MSD is for a $12 \mu \mathrm{m}$ filament $\delta R_{\perp \text {, sat }}^{2} /$ $\delta R_{\| \text {. sat }}^{2} \approx 0.02$. Only for the smallest filament $(L=$ $5.9 \mu \mathrm{m})$ are the out-of-plane contributions sizable, $\delta R_{\perp, \mathrm{sat}}^{2} / \delta R_{\| \text {, sat }}^{2} \approx 0.3$.

[24] R. Everaers et al., Phys. Rev. Lett. 82, 3717 (1999). 